\title{
Abschied von der Gießkanne? Europäische Kohäsionspolitik nach dem Vertrag von Lissabon
}

\author{
Matthias Freise und Matthias Garbert*
}

In der öffentlichen Wahrnehmung spielt die europäische Kohäsionspolitik keine große Rolle. Viel zu komplex ist die Ausgestaltung der Instrumente zur Milderung des strukturellen, wirtschaftlichen und sozialen Ungleichgewichts zwischen den europäischen Regionen, als dass sie es regelmäßig in die Presseberichterstattung schaffen würden. Konkret handelt es sich dabei um den Europäischen Sozialfonds (ESF), den Europäischen Fonds für regionale Entwicklung (EFRE) und den Kohäsionsfonds. Vergegenwärtigt man sich allerdings das finanzielle Volumen der Kohäsionspolitik (bisweilen auch als Struktur- oder Regionalpolitik bezeichnet) mit einem Drittel am Haushalt, wird klar, welche Relevanz das Politikfeld mittlerweile im Tätigkeitsspektrum der Europäischen Union besitzt. So stehen allein im aktuell laufenden vierten Förderzeitraum (2007-2013) 347 Milliarden Euro für die strukturelle Angleichung der europäischen Regionen zur Verfügung. Der Löwenanteil von 81,5 Prozent fließt in die sogenannte Konvergenzförderung, wird also für Regionen bereitgestellt, deren Bruttoinlandsprodukt unter 75 Prozent des EU-Durchschnitts liegt. Dabei profitieren vor allem die mittel- und osteuropäischen Mitgliedstaaten, die 2005 beziehungsweise 2007 der Union beigetreten sind. Gleichwohl werden auch nicht unerhebliche Fördermittel für die regionale Wettbewerbsfähigkeit und Beschäftigung (16 Prozent) sowie für die europäische territoriale Zusammenarbeit (2,5 Prozent) für stärker entwickelte Regionen aufgewendet. ${ }^{1}$ Dies hat der europäischen Kohäsionspolitik zwar seit Jahrzehnten den Vorwurf einer ineffizienten sowie ineffektiven Gießkannenpolitik eingehandelt, ${ }^{2}$ die undifferenziert Fördermittel ausschütte. Anderseits betitelte Ingeborg Tömmel dieses Vorgehen bereits 1994 nicht zu Unrecht als „Joker im politischen Kräftespiel““3 der europäischen Akteure, dient die Kohäsionspolitik doch als Legitimierungsressource für den Integrationsprozess. Diese Diagnose ist in der gegenwärtigen Schuldenkrise aktueller denn je, kann sich die Europäische Union mit ihren Förderprogrammen doch als Innovationsmotor inszenieren und Solidarität in den Mitgliedstaaten leisten, in denen überschuldete Haushalte nationale Wirtschafts- und Sozialpolitik an die Grenzen ihrer Handlungsfähigkeit führen. So hat die Europäische Kommission etwa zusammen mit den griechischen Behörden in den vergangenen Monaten über 180

* Dr. Matthias Freise, Westfälische Wilhelms-Universität Münster.

Matthias Garbert, B.A., Ruprecht-Karls-Universität Heidelberg.

1 Vgl. Europäische Union: Die Kohäsionspolitik 2007-2013. Erläuterungen und offizielle Texte, Luxemburg 2007, S. 10.

2 Vgl. Helen Wallace: The Establishment of the Regional Developement Fund: Common Policy or Pork Barrel?, in: Helen Wallace/William Wallace/Carole Webb (Hrsg.): Policy-Making in the European Communities, London/ New York/Sydney/Toronto 1977, S. 137-163, hier S. 138; vgl. Peter Becker: Die europäische Kohäsionspolitik und ihre Modernisierung, Stiftung Wissenschaft und Politik: SWP-Studien S5/2009, hier S. 29; vgl. Lutz Fischer: Die europäische Strukturpolitik, in: Olaf Leiße (Hrsg.): Governance in Europa. Politikformen im Mehrebenensystem, Berlin 2010, S. 53-69, hier S. 11; vgl. Tanja Kopp-Malek/Marta Lackowska: Structural Funds, in: Hubert Heinelt/Michèle Knodt (Hrsg.): Policies within the EU Multi-Level System. Instruments and Strategies of European Governance, Baden-Baden 2011, S. 153-170, hier S. 153.

3 Ingeborg Tömmel: Staatliche Regulierung und europäische Integration. Die Regionalpolitik der EG und ihre Implementation in Italien, Baden-Baden 1994, S. 17. 
Einzelprojekte initiiert und dabei 11,5 Milliarden Euro mobilisieren können, von denen man sich die Schaffung von 90.000 bis 108.000 neuen Arbeitsplätzen erhofft. ${ }^{4}$

Vor diesem Hintergrund kann auch der Verhandlungsprozess um die Ausrichtung und finanzielle Ausstattung der Instrumente für die kommende Förderperiode (2014-2020) analysiert werden. In der Debatte dominieren dabei drei große Konfliktlinien zwischen Kommission, Parlament und Mitgliedstaaten: Zunächst steht die Frage im Raum, wie umfangreich die Finanzinstrumente in der nächsten Förderperiode ausgestattet werden sollen. Ferner geht es um den konkreten Mitteleinsatz und die Frage, ob und wie die bisherigen Förderschwerpunkte weiterentwickelt werden. Schließlich ist Kohäsionspolitik immer auch Machtpolitik, wird doch auch darum gerungen, ob der Kommission künftig eine größere Steuerungskompetenz bei der Entwicklung der Förderleitlinien zugestanden werden soll, als es bisher der Fall ist.

Wie diese Konflikte letztlich beigelegt werden, lässt sich erst am Ende des Aushandlungsprozesses beurteilen, der maßgeblich durch die Ergebnisse der im November 2012 vorerst gescheiterten Verhandlungen über den mehrjährigen Finanzrahmen der Europäischen Union im Europäischen Rat bestimmt wird. Gleichwohl kann bereits jetzt festgestellt werden, dass der kohäsionspolitische Verhandlungsrahmen durch den Vertrag von Lissabon eine neue Struktur erhalten hat. Wie sich diese Neuerungen auf die Verhandlungen um die künftige Ausgestaltung der Kohäsionspolitik auswirken und welche politischen Inhalte dabei von Kommission, Parlament und Rat der Europäischen Union angestrebt werden, ist Gegenstand der nachfolgenden Analyse. Sie zeigt, dass die europäische Kohäsionspolitik in der bevorstehenden Förderperiode keinen radikalen Strukturbruch, gleichwohl aber weitreichende Modifikationen erfahren wird.

In einem ersten Schritt zeichnet der Beitrag die Entwicklung des Politikfeldes bis zum Vertrag von Lissabon nach, um dann nach den Änderungen der Polity-Strukturen im Vertrag über die Arbeitsweise der Europäischen Union (AEUV) ${ }^{5}$ zu fragen. Sodann werden Konfliktlinien in den aktuellen Verhandlungen herausgearbeitet, um schließlich eine Prognose für die Grundlinien der künftigen Förderprinzipien abzugeben. Im Mittelpunkt steht dabei die Frage, ob eine Abkehr von der viel gescholtenen Gießkannenpolitik zu erwarten ist oder ob die neue Polity-Struktur nicht sogar eine kohäsionspolitische Sprinkleranlage nach sich ziehen wird.

Der Beitrag basiert auf einer umfangreichen Dokumentenanalyse und einer Interviewerhebung mit Expertinnen und Experten aus Parlament, Kommission und Ministerrat sowie aus der erweiterten Systemstruktur, die im Frühjahr und Sommer 2012 durchgeführt worden sind.

\section{Ein Politikfeld im Wandel: Europäische Kohäsionspolitik seit 1988}

„Die Kohäsionspolitik ist die ,sichtbare Hand“ des Marktes.“6 Diese Einschätzung der EU-Kohäsionspolitik durch die ehemalige Kommissarin für Regionalpolitik Danuta Hübner nimmt Bezug auf die Umverteilungs- und Ausgleichsfunktion, die dieses Politikfeld für den EU-Binnenmarkt besitzt. Und auch wenn mit dem 1958 gegründeten ESF die Anfänge der

4 Vgl. Europäische Kommission: Regionalpolitik und die Finanzkrise, abrufbar unter: http://ec.europa.eu/regional_policy/what/crisis/index_de.cfm (letzter Zugriff: 4.2.2013).

5 Vgl. Konsolidierte Fassungen des Vertrags über die Europäische Union (EUV) und des Vertrags über die Arbeitsweise der Europäischen Union (AEUV), in: Amtsblatt der EU, Nr. C 326 vom 26. Oktober 2012.

6 Danuta Hübner: Regionen sind wichtig, in: Panorama-Magazin 26/2008, S. 2-5, hier S. 2. 
Kohäsionspolitik bis zu den Römischen Verträgen zurückreichen, erfolgte die Geburtsstunde dieses Politikfeldes im heutigen Sinne gleichwohl erst drei Jahrzehnte später. Den Hintergrund hierfür bildete die EG-Süderweiterung, das Inkrafttreten der Einheitlichen Europäischen Akte (1987) und das damit verbundene Bestreben, das europäische Binnenmarktprogramm umzusetzen. Dabei wurde schnell klar, dass die Verwirklichung des Gemeinsamen Marktes zwingend nach einer Vergemeinschaftung der Struktur- und Wirtschaftsförderung verlangte. Einerseits mussten Mitgliedstaaten, die Nachteile durch die Verwirklichung des Gemeinsamen Marktes erleben würden, eine Kompensationsperspektive erhalten, andererseits war es ebenso erforderlich den Mitgliedstaaten das Instrument marktverzerrender Wirtschaftsförderung aus der Hand zu nehmen. Dabei ist die Kohäsionspolitik ein Paradebeispiel für Regulierungspolitik im Sinne Giandomenico Majones: ${ }^{7}$ Während die Nationalstaaten auf Vorschlag der Kommission strategische Leitlinien für die Kohäsionspolitik aushandeln und verabschieden, obliegt die konkrete Umsetzung der nationalen strategischen Rahmenpläne und der auf ihnen beruhenden operationellen Programme den Nationalstaaten beziehungsweise den ihnen untergeordneten Ebenen. Der Kommission wird eine Watchdog-Funktion zugewiesen, indem sie die Einhaltung der strategischen Leitlinien überwacht, aber an der Verwirklichung der operationellen Programme selbst nicht mitwirkt. Mit anderen Worten: Der Kommission kommt bei der Programmierung von EFRE, ESF und dem Kohäsionsfonds eine zentrale Rolle zu, während sie beispielsweise bei der Sanierung von Industrieflächen, der Ansiedelung von klein- und mittelständischen Unternehmen (KMU) oder der Errichtung eines Kaltwassergeysirs zur Ankurbelung des Tourismus in der Regel nicht mehr selbst zum Zuge kommt. ${ }^{8}$

Dass das Jahr 1988 für die Kohäsionspolitik eine Zäsur bedeutet, wird allein schon an den strukturellen Voraussetzungen deutlich. So verdoppelte der Rat der Europäischen Union für die erste Förderperiode von 1989 bis 1993 nicht nur die entsprechenden Strukturfondsmittel, sondern es wurden auch zum ersten Mal verbindliche Förderziele eingeführt. Dies ist auch vor dem Hintergrund der anstehenden Währungsunion und dem Maastricht-Vertrag zu sehen, die durch den Abbau weiterer Handelsschranken vor allem die reichen alten Mitgliedstaaten begünstigten und für die Volkswirtschaften der ärmeren Staaten keine substanziellen Vorteile erwarten ließen. So knüpften vor allem Staaten wie Spanien, Portugal und Griechenland aber auch Irland ihre Zustimmung zum Vertragswerk an finanzielle Kompensationsleistungen in Form einer modifizierten und ausgeweiteten Strukturpolitik. ${ }^{9}$ Auch wenn die Kohäsionspolitik bereits zuvor Geber- und Nehmerstaaten kannte, wurde doch erst mit dem Maastricht-Vertrag der finanzielle Rahmen der Kohäsionspolitik so ausgeweitet, dass sich mit Fug und Recht von einer Transferunion sprechen lässt. Freilich war diese nicht auf die Vergemeinschaftung von Schulden gerichtet, wie dies gegenwärtig im Kontext der Euroschuldenkrise diskutiert wird, sondern ein Interessenausgleich zwischen potenziellen Gewinnern und Verlierern einer vertieften Integration.

Dieser bis heute verfolgte Ansatz schlug sich dann vor allem in der großen Erweiterungsrunde 2004/2007 um die mittel- und osteuropäischen Staaten nieder und mittlerweile sind die Struktur- und Kohäsionsfonds mit dem Agrarhaushalt in Hinblick auf das Fördervolumen

7 Giandomenico Majone: The rise of statutory regulation in Europe, in: Giandomenico Majone (Hrsg.): Regulating Europe, Oxford 1996, S. 47-60, hier S. 57.

8 Diese und weitere Beispiele findet man in der Projektdatenbank der Generaldirektion Regionalpolitik, abrufbar unter: http://ec.europa.eu/regional_policy/indexes/project_examples_de.cfm (letzter Zugriff: 30.11.2012).

9 Vgl. Konrad Lammers: Die EU-Regionalpolitik im Spannungsfeld von Integration, regionaler Konvergenz und wirtschaftlichem Wachstum, in: Raumforschung und Raumordnung 4/2007, S. 288-300, hier S. 290. 
nahezu gleichgezogen. Obwohl rein buchhalterische Gegenüberstellungen aufgrund des sehr verschieden ausgeprägten Integrationsnutzens immer problematisch sind, belegt Grafik 1 doch, wie sich die Haushaltssalden der Mitgliedstaaten der Europäischen Union 2011 gestaltet haben. Die Grafik stellt Nettozahler und Nettoempfänger dar, indem sie die Nettozahlungen beziehungsweise Nettoempfänge auf das Bruttoinlandsprodukt der Mitgliedstaaten umrechnet. Sie räumt dabei mit der weit verbreiteten Annahme auf, Deutschland sei ,Zahlmeister Nr. 1' der Europäischen Union, wenngleich sich der Nettobeitrag wegen der vergleichsweise starken deutschen Konjunktur in der Eurokrise tatsächlich vergrößert hat.

\section{Abbildung 1: Operative Haushaltssalden der Mitgliedstaaten der Europäischen Union 2011, Anteil am Bruttoinlandsprodukt in Prozent, Nettobeträge in Milliarden Euro in Klammern}

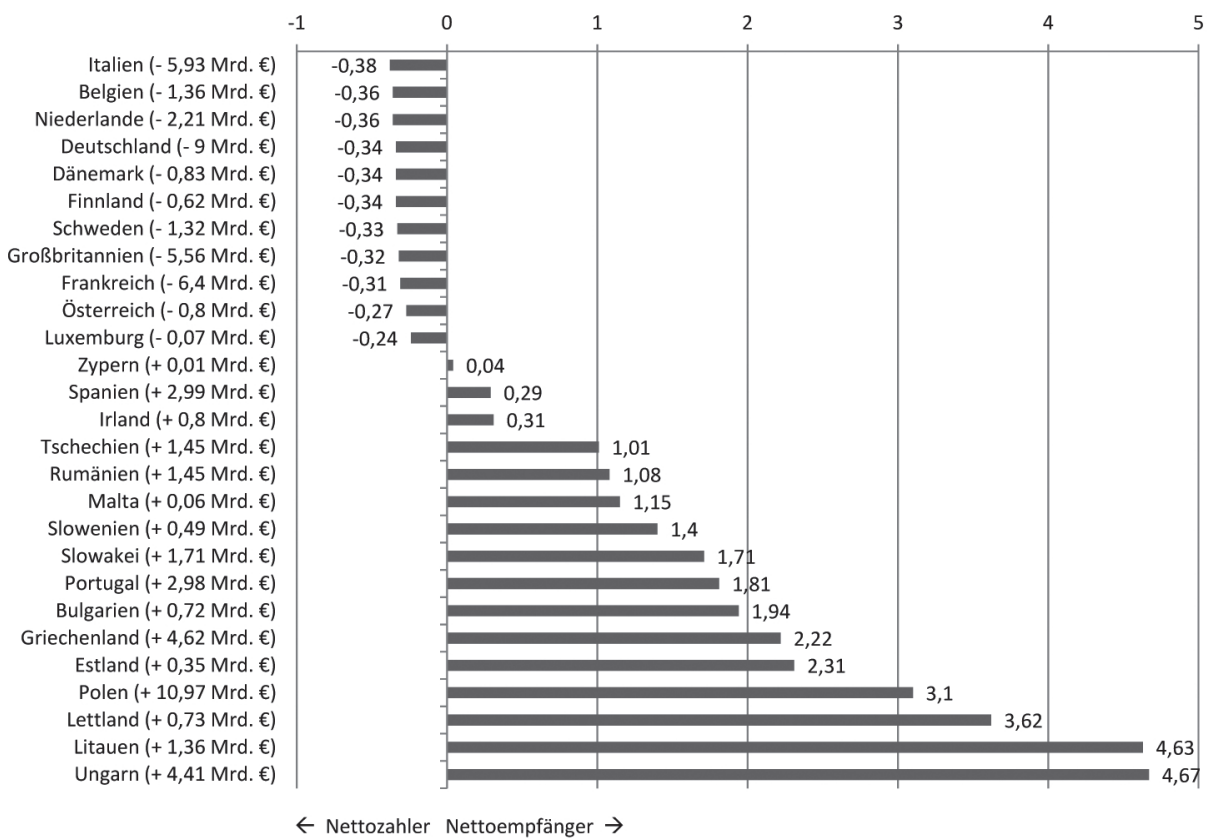

Quelle: Europäische Kommission: EU-Haushalt 2011 - Finanzbericht, Anhang 3.

Mit dem Maastricht-Vertrag installierte man zudem allgemeine Förderprinzipien, die das Politikfeld bis heute prägen. ${ }^{10}$ Konkret handelt es sich hierbei um die Prinzipien der Programmierung, Additionalität, Konzentration und Partnerschaft. ${ }^{11}$ Programmierung meint hierbei, dass die ergriffenen Maßnahmen auf einem strategischen, mehrjährigen Programmansatz statt auf unzusammenhängenden Einzelprojekten beruhen sollen. Die Regel der Additionalität stellt in diesem Kontext sicher, dass die EU-Mittel nationalstaatliche Maßnahmen

10 Vgl. John Bachtler/Carlos Mendez: Who Governs EU Cohesion Policy? Deconstructing the Reforms of the Structural Funds, in: Journal of Common Market Studies 3/2007, S. 535-564, hier S. 537; Heinz-Jürgen Axt: EU-Strukturpolitik. Einführung in die Politik des wirtschaftlichen und sozialen Zusammenhalts, Opladen 2000, S. 59-60; Wolfgang Petzold: 1989-1993: Von Projekten zu Programmen, in: Panorama-Magazin 26/2008, S. 8-14, hier S. 8.

11 Vgl. Sonja Schröder: The 2007-2013 European Cohesion Policy - A New Strategic Approach by the Commission?, Zentrum für Europäische Integrationsforschung: ZEI Discussion Paper C190/2008, S. 1-41, hier S. 4. 
ergänzen statt diese zu ersetzen. Das bedeutet, dass die eingesetzten Fördermittel nur zum Teil aus dem EU-Budget stammen und die Mitgliedstaaten beziehungsweise die ihnen untergeordneten Regionen immer einen Eigenanteil leisten müssen. Der Grundsatz der Konzentration fordert die Fokussierung auf eine begrenzte Anzahl von Zielen, vor allem in den am wenigsten entwickelten Mitgliedstaaten. Das Partnerschaftsprinzip schließlich soll eine zufriedenstellende Partizipation der unterschiedlichen, staatlichen Akteure auf lokaler, nationaler und supranationaler Ebene bei der Planung und Umsetzung der Kohäsionspolitik sicherstellen. ${ }^{12}$ Diese vier Prinzipien dienen jedoch nicht nur der praktischen Umsetzung des europarechtlich fixierten Ziels einer „Politik zur Stärkung [...] wirtschaftlichen, sozialen und territorialen Zusammenhalts“, ${ }^{13}$ sondern können auch als analytische ,Leitplanken“ zur Beschreibung von kohäsionspolitischen Kontinuitäten und Wandlungsprozessen herangezogen werden. So lassen sich beim Vergleich der letzten (2000-2006) mit der derzeitigen Förderperiode (2007-2013) eine Reihe von strategischen Veränderungen, aber auch alt bekannte ,policymaking'-Konzepte feststellen: Beispielsweise hätte 2004 ein konsequentes Festhalten am Prinzip der Konzentration auf die bedürftigsten Regionen eine massive Verschiebung der Finanzzuweisungen zugunsten der neuen Mitgliedstaaten nach sich gezogen und insbesondere in den südeuropäischen Mitgliedstaaten, aber auch in Ostdeutschland für einen Wegfall der Förderprogramme gesorgt. Um die Zustimmung der alten Empfängerstaaten zum Beitritt nicht zu gefährden, wurden großzügige Übergangsregelungen für die Regionen geschaffen, die ohne die Osterweiterung weiterhin förderfähig gewesen wären. ${ }^{14}$ An diesem modus operandi zeigte sich einmal mehr die Schlüsselrolle der Kohäsionspolitik für den europäischen Integrationsprozess: Mittels finanzieller Kompensationen (sogenannten , sidepayments ${ }^{6}$ ) werden potenzielle Integrationsverlierer entschädigt und so die Kompromissfähigkeit einzelner Mitgliedstaaten in Bezug auf Integrationserweiterung und -vertiefung sichergestellt. ${ }^{15}$

Deutliche Neuansätze der Kohäsionspolitik in der aktuellen Förderperiode lassen sich hingegen bei den Prinzipien der Programmierung und Partnerschaft beobachten. Ursächlich hierfür war zum einen die starke programmatische Rückbindung an die Ziele der im Jahre 2000 verabschiedeten Lissabon-Strategie und zum anderen eine Veränderung der Akteurskonstellationen im Bereich der Programmierung. Ersteres spiegelte sich unter anderem in den Programmplanungsdokumenten wider, die zwar weiterhin auch einen territorialen Fokus auf Kohäsion legten, aber ebenso nachdrücklich die Berücksichtigung der sektoralen Ziele der Lissabon-Strategie von Wachstum, Wettbewerbsfähigkeit und Innovation einforderten. ${ }^{16}$ Becker wertet dies als Zielkonflikt und stellt fest: „Die Strukturfonds setzen auf regionale Konvergenz und dezentrale Implementierung, die Lissabon-Strategie hebt auf EUweite Zielvorgaben und eine zentrale Steuerung und Koordinierung der Prozesse ab. "17 Zwar sollten die von Becker genannten Spannungen für das Politikfeld nicht geleugnet werden, gleichwohl lässt sich die Anbindung der Kohäsionspolitik an einen strategischen Rahmenentwicklungsplan, wie sie die Lissabon-Strategie darstellt, auch anders bewerten. So mussten die Reformvorschläge für die Ausgestaltung der aktuellen Förderperiode einer Reihe von

12 Vgl. Bachtler/Mendez: Who Governs EU Cohesion Policy?, 2007, S. 537.

13 Art. 174 AEUV.

14 Vgl. Schröder: The 2007-2013 European Cohesion Policy, 2008, S. 30.

15 Becker: Die europäische Kohäsionspolitik, 2009, S. 56.

16 Vgl. Schröder: The 2007-2013 European Cohesion Policy, 2008, S. 31; Becker: Die europäische Kohäsionspolitik, 2009, S. 16.

17 Becker: Die europäische Kohäsionspolitik, 2009, S. 20. 
Ansprüchen gerecht werden: Beispielsweise gab es vermehrt Kritik der Fachöffentlichkeit an der Wirksamkeit der Kohäsionspolitik, die Verlierer der Osterweiterung hatten Interesse an der Erhaltung des Status quo und schließlich übten die EU-Nettozahler massiven Druck auf den EU-Haushalt aus. ${ }^{18}$ Vor diesem Hintergrund gelang es der Kommission mittels der Anbindung der Kohäsionspolitik an die Lissabon-Strategie Mittelkürzungen abzuwehren, die eigene Position langfristig zu stärken und angesichts struktureller Defizite der europäischen Wirtschaft die Kohäsionspolitik neu zu legitimieren. ${ }^{19}$

Gleichzeitig erfolgten im Bereich der Programmierung massive Veränderungen hin zu einer Stärkung von Dezentralisierung und Subsidiarität. Dies machte sich unter anderem in einem Rückzug der Kommission von der kohäsionspolitischen Implementierungsebene bemerkbar, der begleitet wurde von der Konzentration auf eigene, strategische Kernkompetenzen. ${ }^{20}$ Durch diese Modifikationen im Bereich der Programmierung wurde der Forderung nach einer verwaltungstechnischen Vereinfachung Rechnung getragen, aber auch Spannungen mit den Mitgliedstaaten im Kontext des Partnerschaftsprinzips entschärft. ${ }^{21}$ Dies jedoch als kohäsionspolitische Renationalisierung und Schwächung des Einflusses der Kommission zu deuten, wäre vor dem Hintergrund der Erfahrungen vergangener Förderperioden verfehlt. ${ }^{22}$ Vielmehr führte die Dezentralisierung im Implementationsverfahren der Kohäsionspolitik nicht zu einer Renationalisierung, sondern zu einer stärkeren Europäisierung der Kohäsionspolitik, die auf der gesteigerten politischen Verantwortlichkeit der Mitgliedstaaten für die Implementationsergebnisse beruht. ${ }^{23}$ Betrachtet man diese Reformmaßnahmen in einem breiteren Kontext, scheint es sich um einen politikfeldübergreifenden Trend zu handeln, erlebte doch die EU-Wettbewerbspolitik in der Vergangenheit eine ähnliche steuerungspolitische Neuausrichtung. ${ }^{24}$ Ungeachtet dieser kohäsionspolitischen Neuansätze im Bereich der Grundprinzipien dieses Politikfeldes handelte es sich dabei keineswegs um eine Revolution, sondern vielmehr um pfadabhängige Reformen, ${ }^{25}$ die akteursspezifisch bestimmten Mustern folgten: Während die Kommission die Reformen des Politikfeldes als integrationspolitische Triebfeder nutzen wollte, ${ }^{26}$ versuchte der Rat der Europäischen Union „eine allzu rapide Europäisierung einer Vielzahl von Politiken mit Hilfe der Strukturfonds zu drosseln. “27 Dieses Muster zeigt sich auch nach dem Inkrafttreten des Vertrags von Lissabon.

\section{Kohäsionspolitik nach dem Lissabon-Vertrag}

Die derzeitigen Verhandlungen über die fünfte Förderperiode finden erstmalig unter den Rahmenbedingungen des AEUV statt. Das bedeutet eine ganze Reihe von Neuerungen, ins-

18 Vgl. Wolfgang Petzold: Zur Debatte und den Perspektiven der EU-Strukturpolitik nach 2006, in: Ines Hartwig/ Wolfgang Petzold (Hrsg.): Solidarität und Beitragsgerechtigkeit. Die Reform der EU-Strukturfonds und die Finanzielle Vorausschau, Baden-Baden 2005, S. 117-135, hier S. 134.

19 Vgl. Schröder: The 2007-2013 European Cohesion Policy, 2008, S. 32.

20 Vgl. Kopp-Malek/Lackowska: Structural Funds, 2011, S. 168.

21 Vgl. Schröder: The 2007-2013 European Cohesion Policy, 2008, S. 32

22 Vgl. Bachtler/Mendez: Who Governs EU Cohesion Policy?, 2007, S. 556.

23 Vgl. Schröder: The 2007-2013 European Cohesion Policy, 2008, S. 32.

24 Vgl. Annette E. Töller: Wettbewerbspolitik, in: Hubert Heinelt/Michèle Knodt (Hrsg.): Politikfelder im EUMehrebenensystem. Instrumente und Strategien europäischen Regierens, Baden-Baden 2008, S. 115-139, hier S. 137.

25 Vgl. Fischer: Die europäische Strukturpolitik, 2010, S. 66.

26 Vgl. Ingeborg Tömmel: Neuer Wein in alten Schläuchen? Die Vorschläge der Europäischen Kommission zur Reform der Strukturreform 2007-2013, in: Ines Hartwig/Wolfgang Petzold (Hrsg.): Solidarität und Beitragsgerechtigkeit. Die Reform der EU-Strukturfonds und die Finanzielle Vorausschau, Baden-Baden 2005, S. 57-66, hier S. 65.

27 Ebenda, S. 66. 
besondere in Hinblick auf die Rolle des Europäischen Parlaments. Bis zum Inkrafttreten des Reformvertrags war die Politikarena noch deutlich übersichtlicher und im Wesentlichen Schauplatz von Auseinandersetzungen zwischen Kommission und Ministerrat, wenngleich die Mitgliedstaaten nicht als geschlossene Einheit im Rat auftraten, sondern sich vielmehr in Empfänger- und Geberstaaten unterscheiden ließen. Beispielsweise koordinieren sich bis heute die „like minded states“ Deutschland, Frankreich, Großbritannien, die Niederlande sowie die nordischen Länder und verfechten eine Position, die auf eine Beschränkung der Mittelausstattung ausgerichtet ist, ${ }^{28}$ wobei sich die deutschen Vertreter im Ministerrat in einer Sonderrolle wiederfinden, profitieren doch die neuen Bundesländer bisher auch von den europäischen Fördermitteln. Gleichwohl sind in der Kohäsionspolitik im Rat eine ausgeprägte Konsensorientierung und eine Tendenz zu Paketlösungen zu beobachten, auch wenn der Art. 177 AEUV für die fünfte Förderperiode erstmals das Verfahren der qualifizierten Mehrheit im Rat der Europäischen Union vorsieht. Dabei variieren die ratsinternen Verhandlungsebenen von einfachen Arbeitsgruppen bis hin zu „high-level-groups“, je nach Konfliktgrad der einzelnen Verhandlungsgegenstände. ${ }^{29}$ Um hierbei Selbstblockaden des Rates der Europäischen Union zu verhindern, haben sich beispielhaft im kohäsionspolitischen Bereich verschiedene Strategien herausgebildet, um die Konsensfindung zu erleichtern. Zum einen setzt man auf weit interpretierbare Formulierungen bei der Programmierung der Finanzinstrumente, die zudem idealerweise auch relativ autonomieschonend sind. Weiterhin ist eine Modularisierung von Entscheidungsprozessen zu beobachten, die es ermöglicht, strittige Punkte zunächst auszuklammern. ${ }^{30}$ Dieses Bestreben lässt sich auch in den aktuellen Verhandlungen über den mehrjährigen Finanzrahmen der Europäischen Union beobachten, deren Bedeutung für die zukünftige Ausgestaltung der Kohäsionspolitik in ihrer vorstrukturierenden Funktion liegt. Diese Funktion ist auch charakteristisch für den Europäischen Rat. Er ist dem Rat der Europäischen Union zwar übergeordnet, gleichwohl nicht direkt am EU-Gesetzgebungsverfahren beteiligt. Ungeachtet dessen kann die Versammlung der Staats- und Regierungschefs als Ideengeberin und Weichenstellerin den europäischen Integrationsprozess sowohl bei festgefahrenen Verhandlungen reaktivieren, aber auch allgemein vorstrukturieren. Ein Beispiel hierfür ist ein gemeinsamer Brief von Kanzlerin Merkel und dem ehemaligen französischen Präsidenten Sarkozy an den Präsidenten des Europäischen Rates Van Rompuy, indem es unter anderem hieß: ,Zur Unterstützung notwendiger Reformen sollten die Struktur- und der Kohäsionsfonds genutzt werden, um Wirtschaftswachstum und Wettbewerbsfähigkeit im Euro-Währungsgebiet voranzutreiben. "31 Zwar gab es kommissionsintern gegen die im Brief genannten Vorschläge massiven Widerstand, gleichwohl musste die Kommission aufgrund starken deutsch-französischen Drucks einlenken und es ist sehr wahrscheinlich, dass zumindest der endgültige finanzielle Rahmen für die nächste Förderperiode Anfang 2013 auf einem Treffen des Europäischen Rates ausgehandelt wird. ${ }^{32}$ Dabei werden mit Sicherheit auch noch einmal die Leitlinien der Kohäsionspolitik auf den Prüfstand kommen.

28 Dies ist eines der Ergebnisse der Interviews, die die Autoren im Februar 2012 in Brüssel führten.

29 Vgl. Kopp-Malek/Lackowska: Structural Funds, 2011, S. 164.

30 Ebenda, S. 165.

31 Presse- und Informationsamt der Bundesregierung: Gemeinsamer Deutsch-Französischer Brief an EU-Ratspräsident Herman Van Rompuy, 17.8.2011, Pressemitteilung 291.

32 Vgl. Peter Becker: Lost in Stagnation. Die Verhandlungen über den nächsten mehrjährigen Finanzrahmen der EU (2014-2020) und das Festhalten am Status quo, Stiftung Wissenschaft und Politik: SWP-Studien S 18/2012, S. 9. 
Die Zurückhaltung in der Generaldirektion Regionalpolitik wird verständlich, wenn man die Rolle der Europäischen Kommission in der Kohäsionspolitik näher betrachtet. Für sie ist die Politikarena seit jeher ein wesentlicher, integrationspolitischer Katalysator. So bestätigt ein Blick in die Gesetzgebungsvorschläge der Kommission aus dem Oktober 2011, dass die Kommission eine ganze Reihe von Vorschlägen zur Weiterentwicklung der Kohäsionspolitik vorgelegt hat. Diese laufen im Wesentlichen auf einen Kompetenzzuwachs für die Generaldirektion für Regionalpolitik (Regio) hinaus. Exemplarisch hierfür sind die von der Kommission geforderten Sanktionsmöglichkeiten im Bereich der makroökonomischen Konditionalitäten. ${ }^{33}$

Das Europäische Parlament hingegen wird erst durch den Vertrag von Lissabon zum relevanten Akteur in der kohäsionspolitischen Arena. Beteiligte das Mitentscheidungsverfahren bislang die Parlamentarier lediglich in ausgewählten Politikbereichen, wird das Europäische Parlament mit dem AEUV auch in der Kohäsionspolitik durch dieses Entscheidungsverfahren ${ }^{34}$ aufgewertet. Das heißt, dass sich die Programmierung der Rahmengesetzgebung nicht mehr allein auf Verhandlungen von Mitgliedstaaten und Europäischer Kommission beschränkt, sondern auch die Abgeordneten mit ihren spezifischen Interessen einbinden muss. Insofern ist ein deutlicher Komplexitätszuwachs im Aushandlungsverfahren festzustellen, wie sich auch in der Aufwertung der beiden Parlamentsausschüsse (Ausschuss für regionale Entwicklung (REGI) mit Zuständigkeit für EFRE und den Kohäsionsfonds sowie Ausschuss für Beschäftigung und soziale Angelegenheiten (EMPL) mit Zuständigkeit für ESF) zeigt. Darüber hinaus sieht Art. 314 AEUV auch eine Aufwertung der Budgetfunktion des Europäischen Parlaments vor, das fortan nicht mehr nur bei den nicht-obligatorischen Ausgaben in die Haushaltspolitik eingebunden ist, sondern auch die obligatorischen Ausgaben mitbestimmt. Gleichwohl ist die Vetospieler-Funktion des Europäischen Parlaments in der Kohäsionspolitik auch weiterhin begrenzt, denn einerseits haben seine Mitglieder institutionelle Eigeninteressen und werden daher den EU-Haushalt nicht blockieren, andererseits liegt die Letztentscheidung über die Einnahmen der Europäischen Union weiterhin beim Ministerrat. Gerade in einem redistributiven Politikfeld wie der Kohäsionspolitik ist die Ausgestaltung des finanziellen Förderrahmens deshalb naturgemäß abhängig von dem durch die Mitgliedstaaten festgelegten Mittelvolumen. Oder anders ausgedrückt: Wo nichts zu verteilen ist, können auch keine inhaltlichen Akzente gesetzt werden. ${ }^{35}$

\section{Interessenkonstellationen und Konfliktlinien}

Betrachtet man nun die Konfliktlinien, die sich in der Verhandlungsphase um die fünfte Förderperiode (2014-2020) abzeichneten, so stechen besonders fünf Themenkomplexe hervor, über die gestritten wurde oder noch wird. Konkret geht es dabei zunächst um die (1) Mittelausstattung der einzelnen Fonds sowie (2) die damit verbundene Frage der Mittelbindung an und thematischen Konzentration auf die Ziele der Europa 2020-Strategie. ${ }^{36}$ Dabei steht im Raum, inwieweit das eingesetzte Fördervolumen im Vorhinein für bestimmte

33 Vgl. Europäische Kommission: Kohäsionspolitik 2014-2020 - Investieren in Wachstum und Beschäftigung, Luxemburg 2011, S. 3.

34 Art. 174-178 AEUV.

35 Vgl. Becker: Lost in Stagnation, 2012, S. 9.

36 Europäische Kommission: Mitteilung der Kommission. Europa 2020. Eine Strategie für intelligentes, nachhaltiges und integratives Wachstum, KOM (2010) 2020. 
Schwerpunktsetzungen der Europa 2020-Strategie, wie etwa den Ausbau erneuerbarer Energien oder die Förderung von KMU, reserviert ober ob der Mitteleinsatz nicht besser durch flexible Regelungen den regionalen Besonderheiten angepasst werden soll. Ebenfalls umstritten ist die Frage, (3) inwieweit die Mittelausschüttung an Konditionalitäten gekoppelt werden soll. Zur Debatte stehen dabei ex-ante-, ex-post- und makroökonomische Konditionalitäten. Strittig ist hierbei, ob im Vorfeld der Förderung die aus Sicht der Kommission notwendigen ordnungspolitischen und institutionellen Rahmenbedingungen zur Voraussetzung gemacht werden können und die besonders erfolgreiche Umsetzung der Zielvorgaben künftig honoriert werden soll, indem ein Teil der Fördermittel als leistungsgebundene Reserve zurückgehalten wird. Makroökonomische Konditionalitäten zielen demgegenüber auf die haushalts- und wirtschaftspolitische Disziplinierung der Mitgliedstaaten ab. (4) Ein weiterer großer Streitpunkt ist die Frage, wie in der nächsten Förderperiode mit Regionen umgegangen werden soll, die vor der Osterweiterung der Union zu den wirtschaftlich schwächsten Gebieten gehörten und durch den statistischen Effekt des Beitritts nun aus der Konvergenzförderung herausfallen würden. Hier steht zur Diskussion, ob sogenannte Übergangsregionen eingeführt werden sollen, die das bisher praktizierte Phasing-In- und Phasing-OutVerfahren ersetzen würden. Schließlich steht die Frage nach (5) Sanktionsmöglichkeiten für die Kommission im Raum, wenn Vereinbarungen durch die Mitgliedstaaten beziehungsweise ihre Gliedebenen nicht eingehalten werden.

Ausgangspunkt aller Debatten und den hierzu analysierten Dokumenten sind dabei der konkrete Gesetzgebungsvorschlag, den die Kommission mit dem fünften Kohäsionsbericht im Oktober 2011 vorgelegt hat, ihr Vorschlag für den gemeinsamen strategischen Rahmen aus dem März 2012 sowie die Verhandlungsboxen der jeweiligen Ratspräsidentschaften. ${ }^{37}$

Will man vor dem Hintergrund dieses Kaleidoskops an Verhandlungsfragen nicht den Überblick verlieren, ist es sinnvoll, sich zwei Grundsätze zu vergegenwärtigen. Erstens hat man es bei Kommission, Europäischem Parlament und Ministerrat nicht mit drei monolithischen Verhandlungsblöcken zu tun. Vielmehr verlaufen die Interessenkonstellationen und Konfliktlinien nicht nur institutionenextern, sondern auch institutionenintern höchst heterogen und generieren so eine ganz eigene Dynamik. Zweitens gilt nicht nur im Ministerrat der Satz, „dass nichts vereinbart ist, bis alles vereinbart ist" ${ }^{38}$ Gleichwohl ist es möglich, auf der Grundlage von Positionspapieren, Experteninterviews sowie der inhaltlichen Auswertung der Ministerratssitzungen vom 24. April und vom 16. Oktober 2012 deutliche Präferenzen und Differenzen in Bezug auf die oben ausgeführten Themenkomplexe herauszuarbeiten.

Blickt man zunächst auf die Frage nach der künftigen Mittelausstattung, verläuft die Konfliktlinie dabei auf der einen Seite zwischen Kommission, Europäischem Parlament sowie den Nehmerstaaten (den selbst ernannten „,friends of cohesion policy“) und auf der anderen Seite den Nettozahlern, die sich unter dem Namen ,friends of better spending“ organisieren. Fordern Erstere eine leichte Aufstockung oder zumindest eine Aufrechterhaltung des bisherigen Mittelvolumens, wollen die Geberstaaten das Fördervolumen deutlich reduziert wissen, allen voran der britische Premierminister David Cameron, der von seiner Conservative Party erheblich unter Druck gesetzt wurde, das Haushaltsvolumen der Europäischen Union zu

37 Beide fußen wiederum auf dem fünften Kohäsionsbericht, der zunächst die Errungenschaften der vierten Förderperiode bilanziert und daraus Schlussfolgerungen für die Zukunft der Förderpolitik zieht.

38 Rat der Europäischen Union: Mehrjähriger Finanzrahmen (2014-2020) - Verhandlungsbox, Dok. 10063/1/12, S. 1. 
drücken und dabei auch die beiden größten Haushaltsposten, die Agrar- und Kohäsionspolitik, anzugehen. Gleichwohl lässt sich das Bild auch hier differenzieren. So will die Kommission die Mittel in etwa aufrechterhalten, während der Parlamentsausschuss im sogenannten Pieper-Report eine deutliche Mittelaufstockung im Bereich von Wettbewerbsfähigkeit, Beschäftigung und der europäischen territorialen Zusammenarbeit fordert. ${ }^{39}$ Die von der zypriotischen Ratspräsidentschaft und vom Präsidenten des Europäischen Rates Van Rompuy im Vorfeld des gescheiterten EU-Gipfels im November 2012 vorgelegten Kompromissvorschläge sahen hingegen nur leichte Budgeterhöhungen vor, die inflationsbereinigt moderate Kürzungen bei der Ausstattung der Kohäsionspolitik bedeuten würden. Tabelle 1 stellt die aktuelle Mittelausstattung der vierten Förderperiode mit den eingebrachten Vorschlägen für die fünfte Förderperiode gegenüber. Eine abschließende Entscheidung über den mehrjährigen Finanzrahmen der Europäischen Union fällt allerdings vermutlich erst im Frühjahr 2013 im Rahmen eines weiteren Gipfeltreffens der Staats- und Regierungschefs. ${ }^{40}$

Tabelle 1: Aktuelles Kohäsionsbudget und Vorschläge für die Mittelausstattung der 5. Förderperiode (2014-2020)

\begin{tabular}{cccc}
$\begin{array}{c}\text { Aktuelles Bud- } \\
\text { get der 4. För- } \\
\text { derperiode }\end{array}$ & $\begin{array}{c}\text { Kommissionsvor- } \\
\text { schlag vom } \\
\mathbf{2 0 0 7 - 2 0 1 3 )}\end{array}$ & $\begin{array}{c}\text { Kompromissvor- } \\
\text { schlag der zyprioti- } \\
\text { schen Ratspräsident- } \\
\text { schaft vom }\end{array}$ & $\begin{array}{c}\text { Kompromissvor- } \\
\text { schlag des Präsiden- } \\
\text { ten des Europäischen } \\
\text { Rates vom 23.11.2012 }\end{array}$ \\
\hline 308 Mrd. Euro & 376 Mrd. Euro & 363 Mrd. Euro & $311-317$ Mrd. Euro \\
\hline
\end{tabular}

Quelle: Europäische Kommission: Mehrjähriger Finanzrahmen 2014-2020, Dokumentensammlung zum Mehrjährigen Finanzrahmen 2014-2020, abrufbar unter: http://ec.europa.eu/budget/mff/index_de.cfm (letzter Zugriff: 4.2.2013).

Ein zweiter Blick lohnt sich auch auf die Verhandlungen über die künftige Mittelbindung an und thematische Konzentration auf die Ziele der Europa 2020-Strategie. Im Grundsatz standen Ministerrat und Europäisches Parlament diesem Vorhaben der Kommission zustimmend gegenüber. Gleichwohl gab es Dissens über die Schwerpunktsetzung. Während der Kommissionsvorschlag eine möglichst starke und umfassende Verankerung der EU 2020Ziele in allen Fonds vorsah, ${ }^{41}$ begrüßte das Europäische Parlament zwar den Einsatz von Kohäsionsmitteln für die Umsetzung der Europa 2020-Ziele, unterstrich jedoch, ,dass die Kohäsionspolitik nicht nur ein Umsetzungsinstrument für die Strategie Europa 2020 ist. “42 Allerdings wurde dabei parlamentsintern um die Frage gerungen, worauf sich die thematische Konzentration fokussieren sollte und welche Förderschwerpunkte am effektivsten Wachstum und Beschäftigung fördern können. Einig waren sich die Parlamentarier hingegen

39 Vgl. Europäisches Parlament: Bericht über den Fünften Kohäsionsbericht der Kommission und zur Strategie für die Kohäsionspolitik nach 2013 (2011/2035(INI)), Ausschuss für regionale Entwicklung, Berichterstatter: Markus Pieper, 6.6.2011, Dok. A7-0222/2011, S. 14 (Im Folgenden zitiert als Pieper-Report, 2011); Europäische Kommission: Vorschlag für eine Verordnung des Europäischen Parlaments und des Rates mit gemeinsamen Bestimmungen über den Europäischen Fonds für regionale Entwicklung, den Europäischen Sozialfonds, den Kohäsionsfonds, den Europäischen Landwirtschaftsfonds für die Entwicklung des ländlichen Raums und den Europäischen Meeres- und Fischereifonds, für die der Gemeinsame Strategische Rahmen gilt, sowie mit allgemeinen Bestimmungen über den Europäischen Fonds für regionale Entwicklung, den Europäischen Sozialfonds und den Kohäsionsfonds und zur Aufhebung der Verordnung (EG) Nr. 1083/2006 des Rates, KOM (2011) 615, S. 7 (Im Folgenden zitiert als Vorschlag Gemeinsamer Strategischer Rahmen, 2012).

40 Vgl. Europäischer Rat: Erklärung der Mitglieder des Europäischen Rates 23. November 2012, 23.11.2012. S. 1.

41 Vgl. Art. 9 und Art. 81 Abs. 1 des Vorschlags eines Gemeinsamen Strategischen Rahmens, 2012.

42 Vgl. Ziffer 9 Pieper-Report, 2011. 
weitgehend bei der Ablehnung der von der Kommission vorgeschlagenen Höhe der Mittelbindung. ${ }^{43}$ So forderte die Kommission beispielsweise für die EFRE-Mittel, dass in Übergangsregionen beziehungsweise stärker entwickelten Regionen 80 Prozent des Fördervolumens ,auf nationaler Ebene für die Bereiche Energieeffizienz und erneuerbare Energien, Innovation und KMU-Förderung verwendet werden" $" 44$ sollten. Regionen im Bereich der Konvergenzförderung sollten demgegenüber zwar weniger, aber immerhin noch 50 Prozent der EFRE-Mittel für die genannten Ziele ausgeben müssen. ${ }^{45}$ Hier forderte das Europäische Parlament eine massive Senkung der von der Kommission vorgeschlagenen Quoten. ${ }^{46}$ Unterstützung fand es mit dieser Forderung beim Ministerrat, in dem viele Staaten eine ,Einheitsquote' in Bezug auf die differenzierten Bedürfnisse der unterschiedlichen Regionen kritisch sahen. Da jedoch die Fokussierung auf die Europa 2020-Ziele im Grundsatz bei den Verhandlungsakteuren nicht zur Disposition stand, ließ sich im Rat der Europäischen Union in Bezug auf die Quotierung eine aufschlussreiche Kompensationsstrategie beobachten. Statt die Quotierung prinzipiell zu verwerfen, versuchten die Ländervertreter den Katalog der Fördermaßnahmen, die sich unter eine Zielquotenvorgabe subsumieren lassen, massiv auszuweiten. Ist diese Strategie erfolgreich, wäre beispielsweise in der neuen Förderperiode auch der Ausbau von Verkehrswegen unter einer weit gefassten Energieeffizienz-Kategorie förderfähig. ${ }^{47}$ Dies würde je nach Sichtweise der thematischen Konzentration einen Teil ihrer regionalen Inflexibilität beziehungsweise Effektivität nehmen.

Ein weiterer umstrittener Punkt war die Ausgestaltung von Konditionalitäten in der neuen Förderperiode, wenngleich die Zustimmung zu den von der Kommission vorgeschlagenen ex-ante-, ex-post- und makroökonomischen Konditionalitäten stark variierte. So fanden die Vorschläge zur Weiterentwicklung von ex-ante-Konditionalitäten im Europäischen Parlament breite Zustimmung ${ }^{48}$ und riefen auch im Ministerrat keine massive Kritik hervor, ganz im Gegensatz zur Debatte um die Einführung von ex-post-Konditionalitäten. Gegenwärtig werden hierzu im Ministerrat zwei Möglichkeiten diskutiert. Die erste entspricht dabei weitestgehend dem Kommissionsvorschlag, während die zweite vorsieht, die Einrichtung von Leistungsreserven den jeweiligen Nationalstaaten zu überlassen. ${ }^{49}$

Durchaus umstritten waren auch die von der Kommission vorgeschlagenen makroökonomischen Konditionalitäten. Dieser Verhandlungsgegenstand war gerade in Hinblick auf die institutioneninternen Auseinandersetzungen besonders aufschlussreich. Bei einem vordergründigen Blick auf die ausgewerteten Dokumente schienen die Positionen zwischen Kommission und Europäischem Parlament zunächst eindeutig. ${ }^{50}$ Ein solches Schwarz-WeißBild wäre jedoch zu einfach gezeichnet. Kommissionsintern hätte die Generaldirektion für Wirtschaft und Finanzen mittels makroökonomischer Konditionalitäten ein erhebliches Drohpotenzial zur Einforderung haushaltspolitischer Disziplin erhalten. Dass eine solche Instrumentalisierung nicht im Interesse der Generaldirektion für Regionalpolitik lag, resul-

43 Dies ist eines der Ergebnisse der Interviews, die die Autoren im Februar 2012 in Brüssel führten.

44 Europäische Kommission: Kohäsionspolitik 2014-2020, 2011, S. 8.

45 Ebenda.

46 Dies ist eines der Ergebnisse der Interviews, die die Autoren im Februar 2012 in Brüssel führten.

47 Dies ist eines der Ergebnisse der Interviews, die die Autoren im Februar 2012 in Brüssel führten.

48 Vgl. Art. 17 des Vorschlags eines Gemeinsamen Strategischen Rahmens, 2012; vgl. Ziffer 64 Pieper-Report, 2011, S. 19

49 Rat der Europäischen Union: Multiannual Financial Framework (2014-2020) - Negotiating Box, Dok. 11539/12, S. 31 .

50 Vgl. Art. 21 des Vorschlags eines Gemeinsamen Strategischen Rahmens, 2012; Ziffer 64 Pieper-Report, 2011, S. 19. 
tiert aus den möglichen Konflikten zwischen mitgliedstaatlicher Haushaltspolitik und regionaler Förderbedürftigkeit. Das Argument, dass Regionen nicht für eine problematische Haushaltspolitik ihrer Nationalstaaten zur Rechenschaft gezogen werden sollten, fand dabei auch breiten Widerhall im Europäischen Parlament. ${ }^{51}$ Dies ist im Ministerrat nicht der Fall. $\mathrm{Zu}$ deutlich zeichnen sich hierzu die Standpunkte der einzelnen Mitgliedstaaten ab, die in der Regel einer Dichotomie zwischen Geber- und Nehmerstaaten folgen. ${ }^{52}$ Waren Nehmerstaaten wie Spanien, Griechenland oder Polen klar gegen makroökonomische Konditionalitäten, drängten Geberstaaten wie Frankreich, Schweden oder die Niederlande auf ihre Einführung. ${ }^{53}$ Ein ähnlich zweigeteiltes Bild fand sich dort auch bei der Frage nach der Einführung von Übergangsregionen. Hier verliefen die Interessengegensätze jedoch nicht entlang von Geber- und Nehmerstaaten, sondern spalteten Profiteure beziehungsweise Nicht-Profiteure der Einführung einer solchen Förderkategorie in zwei Lager. Waren Geberländer, aber Nicht-Profiteure (vor allem die Niederlande) gegen die Einführung von Übergangsregionen, sah dies bei Geberländern, die zugleich Profiteure dieser Kategorie sind, anders aus. Beispielhaft kann hier auf Schweden und Frankreich verwiesen werden. ${ }^{54}$ Weitgehende Einigkeit, Übergangsregionen einzuführen, herrschte hingegen zwischen Kommission und Europäischem Parlament. ${ }^{55}$ Die geschickte Allianzbildung zwischen Kommission und Abgeordnetenhaus stellt jedoch noch keine Garantie für die tatsächliche Einführung dieser Kategorie dar. Vielmehr ist es sehr wahrscheinlich, dass bei den anstehenden Verhandlungen über den mehrjährigen Finanzrahmen die Ausweitung der Förderregionen noch Gegenstand harter Verhandlungen auf höchster Ebene sein wird - nicht zuletzt vor dem Hintergrund der anhaltenden Schuldenkrise. ${ }^{56}$

Fragt man schließlich nach den Sanktionsmöglichkeiten, die der Kommission für die neue Förderperiode zur Verfügung gestellt werden sollen, wird vor allem um die Möglichkeit der Mittelaussetzung beziehungsweise -streichung gerungen. Da diese Frage unter anderem auch stark mit dem Streitpunkt der makroökonomischen Konditionalitäten verwoben ist, wundert es nicht, dass die Konfliktlinien hier ebenfalls ähnlich verlaufen.

\section{Wohin steuert die europäische Kohäsionspolitik?}

Welche Prognosen lassen sich aus der oben ausgeführten Konfliktlinienanalyse für die europäische Kohäsionspolitik ab 2014 ableiten? Mit großer Wahrscheinlichkeit wird der eigentlich für Anfang 2014 vorgesehene Programmstart der fünften Förderperiode durch das Scheitern des Ratsgipfels im November 2012 nicht termingerecht erfolgen können, sodass die europäische Kohäsionspolitik im Jahr 2014 zunächst unter den alten Rahmenbedingungen weitergeführt wird. Voraussichtlich werden sich Geber- und Nehmerstaaten jedoch auf einem weiteren Ratsgipfel in Bälde doch noch auf einen mehrjährigen Finanzrahmen einigen, in dem die Mittel für die Kohäsionspolitik moderat zurückgefahren werden. Drastische Einschnitte sind angesichts der vertraglichen Rahmenbedingungen nicht zu erwarten, denn blockieren die „friends of cohesion policy“ den neuen Budgetansatz durch ihr Veto, kommt es

51 Dies ist eines der Ergebnisse der Interviews, die die Autoren im Februar 2012 in Brüssel führten.

52 Vgl. Council of the European Union - General Affairs: Legislative deliberations, 2012, abrufbar unter: http:// www.consilium.europa.eu/council/open-sessions/previous-open-sessions/general-affairs?lang=en (letzter Zugriff: 4.2.2013).

53 Ebenda.

54 Ebenda.

55 Vgl. Art. 82 des Vorschlags eines Gemeinsamen Strategischen Rahmens, 2012; Ziffer 34 Pieper-Report, 2011.

56 Dies ist eines der Ergebnisse der Interviews, die die Autoren im Februar 2012 in Brüssel führten. 
bis zu einer Einigung zu einer Übertragung des Haushaltsansatzes aus dem letzten Siebenjahresplan (plus Inflationsausgleich), die die Geberländer letztlich teurer zu stehen käme als eine Zustimmung zu moderaten Einsparungen im Agrar- und Kohäsionsbudget. Die Prognose ist daher nicht allzu gewagt, dass sich der neue mehrjährige Finanzrahmen in etwa am Kompromissvorschlag vom Präsidenten des Europäischen Rates Van Rompuy orientieren wird, der 311 Milliarden Euro für die Kohäsionspolitik ansetzt und in den Jahren 2017 bis 2020 zusätzliche Ausgleichzahlungen in Höhe von weiteren sechs Milliarden Euro in Aussicht stellt. ${ }^{57}$

Völlig unzweifelhaft ist dagegen bereits jetzt, dass die Förderung der Regionen zukünftig wesentlich stärker als bisher an einer überwölbenden Strategie ausgerichtet wird. Bei allen beteiligten Akteuren besteht Einigkeit, dass sich die europäische Kohäsionspolitik der nächsten Jahre an den Kernzielen der Europa 2020-Strategie - Beschäftigung, Innovation, Bildung, soziale Integration sowie Klima/Energie - ausrichten sollte und dass dies eine verstärkte Zentralisierung der Förderprogramme in der Hand der Europäischen Kommission zur Folge haben wird. Wie sehr die Europäische Kommission den Regionen künftig programmatische Vorgaben für die konkrete Umsetzung machen darf und inwieweit tatsächlich eine thematische Mittelbindung der Fonds erfolgen wird, ist gegenwärtig noch nicht abzusehen. Voraussichtlich werden sich die Mitgliedstaaten aber erfolgreich dagegen wehren können, an die Kandare der Kommission genommen zu werden. Wahrscheinlich ist eine Einigung, die die Mittel thematisch an die strategischen Leitlinien der Europa 2020-Strategie bindet, diese jedoch lockerer auslegt, sodass sie Mitgliedstaaten und Regionen ihren Besonderheiten anpassen können.

Sehr unwahrscheinlich ist auch, dass sich die Kommission mit ihren weitreichenden Forderungen nach einer Disziplinierung der künftigen Kohäsionspolitik durch die Verschärfung beziehungsweise Einführung von Konditionalitäten wird durchsetzen können. Insbesondere die Etablierung von makroökonomischen und ex-post-Konditionalitäten scheint gegenwärtig ausgeschlossen. Gegen ex-post-Konditionalitäten wird nicht zu Unrecht die Befürchtung ins Feld geführt, dass Regionen nur noch vermeintlich sichere Investitionsprogramme auflegen werden, wenn sie befürchten müssen, dass eine Zielverfehlung zur Streichung von Fördermitteln führen kann. Ökonomisch sinnvolle, aber nur schwer abzuschätzende Programme würden vermutlich nicht mehr angepackt. Makroökonomische Konditionalitäten würden dagegen eine Bestrafung der Regionen für ein Versagen ihrer Nationalstaaten nach sich ziehen, was besonders in Zeiten der Schuldenkrise der Eurostaaten die Kohäsionspolitik ad absurdum führen würde. Es ist deshalb absehbar, dass der Kommission für den nächsten Förderzeitraum in stärkerem Maße Mitspracherechte bei der Programmierung der Fördermaßnahmen eingeräumt werden, darüber hinaus reichende Konditionalitäten hingegen wahrscheinlich keine Zustimmung im Rat der Europäischen Union und im Europäischen Parlament finden.

Besonders spannend ist schließlich die Frage, die den vorliegenden Beitrag übertitelt: Wird sich europäische Kohäsionspolitik in der anstehenden Förderperiode von der viel kritisierten Gießkannenpolitik verabschieden oder diese sogar ausbauen? Da Kohäsionspolitik in der Europäischen Union immer auch Kompromisspolitik ist, ist eine Beibehaltung der Gießkannenstrategie abzusehen, wenngleich der Gießkannenkopf etwas verengt wird. In anderen Worten: Erwartbar ist eine stärkere Ausrichtung der Kohäsionspolitik auf die Kon-

57 Für einen Überblick über die Choreografie europäischer Budgetverhandlungen vgl. Becker: Lost in Stagnation, 2012. 
vergenzregionen bei gleichzeitiger Einführung von Übergangsregionen (75 bis 90 Prozent des europaweiten durchschnittlichen Bruttoinlandsprodukts) und der Beibehaltung von Förderprogrammen auch für Regionen, die im Grunde keiner Förderung mehr bedürfen. Nur wenn sichergestellt wird, dass Wirtschaftsfördermittel auch künftig in alle Regionen fließen, kann die Kohäsionspolitik weiterhin als Legitimierungsressource des Gemeinsamen Marktes herangezogen werden. In Zeiten, in denen sich die Europäische Union immer vehementer dem Vorwurf mangelnder Legitimität ausgesetzt sieht, ist das ein zu hohes Gut, als dass es zugunsten von Wirtschaftlichkeitsrechnungen aufgegeben würde.

\section{Euro- und Schuldenkrise}

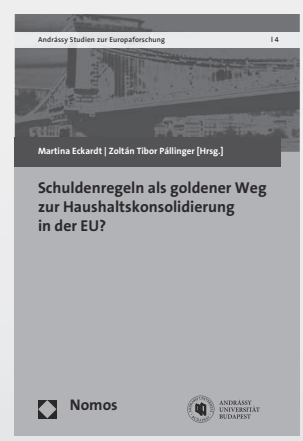

Die Rettung systemrelevanter Banken und internationaler Finanzinstitute hat für viele Staaten der Eurozone zu einem weiteren massiven Anstieg ihrer hohen Verschuldung geführt. Der Band präsentiert eine umfassende,
Schuldenregeln als goldener Weg zur Haushaltskonsolidierung in der EU?

Herausgegeben von Martina Eckardt und Zoltán Tibor Pállinger 2013, ca. 240 S., brosch., ca. 39,-€ ISBN 978-3-8487-0072-1

(Andrássy Studien zur Europaforschung, Bd. 4)

Erscheint ca. Mai 2013

interdisziplinäre Bestandsaufnahme des Instrumentariums zu Schuldenregeln und die Bewertung ihres Potentials zur Lösung der gegenwärtigen Euro- und Schuldenkrise.

\section{Nomos}

